\title{
A pivot mutation impedes reverse evolution across an adaptive landscape for drug resistance in Plasmodium vivax
}

\author{
C. Brandon Ogbunugafor ${ }^{1,2^{*}}$ and Daniel Hartl $\left.\right|^{3}$
}

\begin{abstract}
Background: The study of reverse evolution from resistant to susceptible phenotypes can reveal constraints on biological evolution, a topic for which evolutionary theory has relatively few general principles. The public health catastrophe of antimicrobial resistance in malaria has brought these constraints on evolution into a practical realm, with one proposed solution: withdrawing anti-malarial medication use in high resistance settings, built on the assumption that reverse evolution occurs readily enough that populations of pathogens may revert to their susceptible states. While past studies have suggested limits to reverse evolution, there have been few attempts to properly dissect its mechanistic constraints.

Methods: Growth rates were determined from empirical data on the growth and resistance from a set of combinatorially complete set of mutants of a resistance protein (dihydrofolate reductase) in Plasmodium vivax, to construct reverse evolution trajectories. The fitness effects of individual mutations were calculated as a function of drug environment, revealing the magnitude of epistatic interactions between mutations and genetic backgrounds. Evolution across the landscape was simulated in two settings: starting from the population fixed for the quadruple mutant, and from a polymorphic population evenly distributed between double mutants.
\end{abstract}

Results: A single mutation of large effect (S117N) serves as a pivot point for evolution to high resistance regions of the landscape. Through epistatic interactions with other mutations, this pivot creates an epistatic ratchet against reverse evolution towards the wild type ancestor, even in environments where the wild type is the most fit of all genotypes. This pivot mutation underlies the directional bias in evolution across the landscape, where evolution towards the ancestor is precluded across all examined drug concentrations from various starting points in the landscape.

Conclusions: The presence of pivot mutations can dictate dynamics of evolution across adaptive landscape through epistatic interactions within a protein, leaving a population trapped on local fitness peaks in an adaptive landscape, unable to locate ancestral genotypes. This irreversibility suggests that the structure of an adaptive landscape for a resistance protein should be understood before considering resistance management strategies. This proposed mechanism for constraints on reverse evolution corroborates evidence from the field indicating that phenotypic reversal often occurs via compensatory mutation at sites independent of those associated with the forward evolution of resistance. Because of this, molecular methods that identify resistance patterns via single SNPs in resistanceassociated markers might be missing signals for resistance and compensatory mutation throughout the genome. In these settings, whole genome sequencing efforts should be used to identify resistance patterns, and will likely reveal a more complicated genomic signature for resistance and susceptibility, especially in settings where anti-malarial medications have been used intermittently. Lastly, the findings suggest that, given their role in dictating the dynamics of evolution across the landscape, pivot mutations might serve as future targets for therapy.

*Correspondence: bogbunug@uvm.edu

1 Department of Biology, University of Vermont, Burlington, VT, USA

Full list of author information is available at the end of the article 
Keywords: Plasmodium vivax, Pyrimethamine resistance, Adaptive trajectories, Reverse evolution, Gene by environment interactions

\section{Background}

In recent years, experts have introduced several new perspectives on the management of drug resistance in malaria and other infectious diseases. These include criticisms of the aggressive use of therapeutic agents [1-3], the broader encouragement of more responsible use of antimicrobials [4-7] and the exploration of drug cycling strategies [8-13]. Drug stewardship programmes have been successful in several settings, and declines in drug resistance have been observed following changes in antibiotic use [14, 15]. There are other settings, however, where more careful use of antibiotics was not so effective, microbial populations remaining highly resistant even after removal of drug [16-19], an outcome with serious health and financial consequences. Pathogens might remain resistant to antimicrobials even after their removal for several reasons, among them compensatory mutations at other loci that counteract any fitness cost of drug resistance [18, 20, 21]. While compensatory mutations at other loci underlie many long-term fixation patterns in clinical infections, it is not fully understood why compensatory mutation is necessary, rather than the evolutionary undoing of mutations that 'fixed' in the process of forward resistance evolution.

The lack of a coherent understanding of reverse evolution is partly due to conceptual ambiguity: the term 'reverse evolution' is misleading, as it implies directionality in a process (Darwinian evolution) that is near-sighted and agnostic with regard to goal. This has spawned similarly dubious concepts, such as Dollo's Law, asserting that evolution is intrinsically irreversible [22] because it would require two independent, low-probability events, occurring along the same pathway, but in opposite order [23]. Consequently, few studies have examined the molecular pathways through which reverse evolution across an antimicrobial resistance adaptive landscape is likely to occur. One such study of cefotaxime/pipericillin resistance in Escherichia coli highlighted that epistasis may wire 'hidden randomness' into adaptive landscapes that prevents reverse evolution [24]. A landmark study of reverse evolution in the vertebrate glucocorticoid receptor identified a combination of five mutations, labelled an 'epistatic ratchet', that precludes evolution towards the ancestral state [25]. Studies of this sort are even less frequent as they pertain to the problem of malaria drug resistance, which remains the cause of a global pandemic complicated by widespread resistance [26].
Approaches utilizing all possible combinations of a suite of mutations associated with resistance can help to resolve the likelihood of adaptive evolution occurring through certain pathways [27-31]. This study uses empirical data from a combinatorial analysis of Plasmodium vivax dihydrofolate reducatase (DHFR) mutants, evolutionary theory, and individual-based simulations to uncover factors that affect the likelihood of reverse evolution across pyrimethamine (PYR) concentrations. In doing so, it proposes a method for determining whether reverse evolution will occur across an adaptive landscape. By measuring the fitness effects of individual mutations, the study uncovers the existence of a mutational pivot with potentiated genotype-by-environment $(G \times E)$ effects that may direct evolution towards or constrain evolution from areas of the landscape with high resistance or fitness. In addition, these mutations attract interactions with other mutation sites, creating an epistatic ratchet, limiting reverse evolution across a landscape. Lastly, the study discusses the implications of these findings for evolutionary theory, molecular epidemiology and in two clinically relevant contexts: (1) the use of existing drugs for resistance management in malaria, and, (2) the rational design of drugs that might target certain amino acid residues of a resistance determinant.

\section{Methods}

\section{System of study and growth rates}

The study modelled empirical growth and resistance $\left(\mathrm{IC}_{50}\right)$ data developed in a prior study [32] in strains of transgenic Saccharomyces cerevisiae carrying $P$. vivax DHFR containing a set of four mutations orthologous to the resistance mutations found in Plasmodium falciparum [30], in all combinations, several of which have been isolated from field settings [33-46]. The combinatorial approach is an effective way to create empirical adaptive landscapes for final phenotypes when all intermediate genotypes can be reconstructed in the protein of interest, often in a transgenic setting (Saccharomyces cerevisiae in this case). Because of this, this approach is not meant to be a literal analogue for drug treatment, but does effectively test important properties of protein evolution. Results derived from prior studies of this kind have recapitulated findings from the field [29, 32], reaffirming that this approach has utility in understanding the evolution of drug resistance. 
This study used bit-string notation, with 0 corresponding to the presence of the ancestral state mutation, and 1 a replacement of a mutation observed to confer varying levels of fitness in the presence of drug concentrations. The individual amino acid sites are N50I $\left(1^{* * * *}\right)$, S58R $\left({ }^{*} 1^{* *}\right)$, S117N $\left(^{* * *} 1 *\right)$, and I173L (***1). A diagram of the possible evolutionary trajectories from the state 1111 to 0000 is shown in Fig. 1a.

A logistic growth equation was used to model growth rates across a range of concentrations (Fig. 1b). The growth equation, as described in Jiang et al. [32] is as follows:

$$
g(x)=\frac{g_{\text {drugless }}}{1+e^{\frac{I_{50}-x}{c}}}
$$

where $g_{\text {drugless }}$ is the growth rate in the presence of no drug, the $\mathrm{IC}_{50}$ value in $\mu \mathrm{M}$ and the $c$ a constant that determines the slope of the curve. The estimated growth rates are robust with regard to estimation error, as the standard errors of the estimated $\mathrm{IC}_{50}$ parameters are quite low, generally less than $10 \%$ of the mean (Additional file 1). The growth rates in the study are determined across a broad range (Fig. 1b, Additional file 2) which includes those concentrations of PYR observed in the blood of persons treated with PYR [47-51]. The study used these growth rates to determine the accessibility of pathways, as in prior studies. In order to identify an accessible pathway, the rank orders between alleles must decrease from step-to-step, indicating that a mutation is moving to a higher fitness portion of the landscape (a ranking of 1 meaning the most fit allele in the landscape). Figure 1c demonstrates how the rank order of fitnesses changes as a function of drug concentration (values in Additional file 3). For the purposes of this study, one should note how often the lines cross one another at different drug concentrations. This indicates the presence of gene by environment $(G \times E)$ interactions that alter the structure of the adaptive landscape and create different evolutionary dynamics in different drug environments.

\section{Fitness effect of mutations}

To estimate the interaction between the effect of mutation and drug concentration ( $\mathrm{G} \times \mathrm{E}$ interaction), the effect of individual mutations across drug concentrations was calculated [52]. For the P. vivax DHFR landscape, each mutant site has eight possible genetic backgrounds to which it could be added. To calculate the effect of a mutation, take the difference between the fitness $(W)$ of an allele $j$ and the one-step neighbour carrying mutation $\varepsilon$, where $\varepsilon$ corresponds to mutations: N50I $\left(1^{* * *}\right)$, S58R $\left({ }^{*} 1^{* * *}\right), S 117 \mathrm{~N}\left({ }^{* * *} 1 *\right)$, and I173L $\left(^{* * * *} 1\right)$ :

$$
\Delta W_{\varepsilon}=W_{j}-W_{j_{\varepsilon}}
$$

This was calculated for each of the four mutations across a range of drug concentrations (between 0 and approximately $8000 \mu \mathrm{M}$ ). After calculating the fitness effect of mutations across drug concentrations, the average fitness of all whole alleles carrying each of the four mutations $\left(1^{* * *}, 1^{* * * * * * 1 *} 1^{* * * * 1)}\right.$ ) was measured and compared using ANOVA to determine any significant differences between mutant classes (Additional file 4).

\section{Measuring epistasis}

Embedded in Fig. 3a is epistasis, or the "surprise at the phenotype when mutations are combined, given the constituent mutations' individual effects" [52]. Epistasis was measured by calculating the standard deviation of the total fitness effects for a mutation at a given concentration. These values were plotted in Additional file 5.

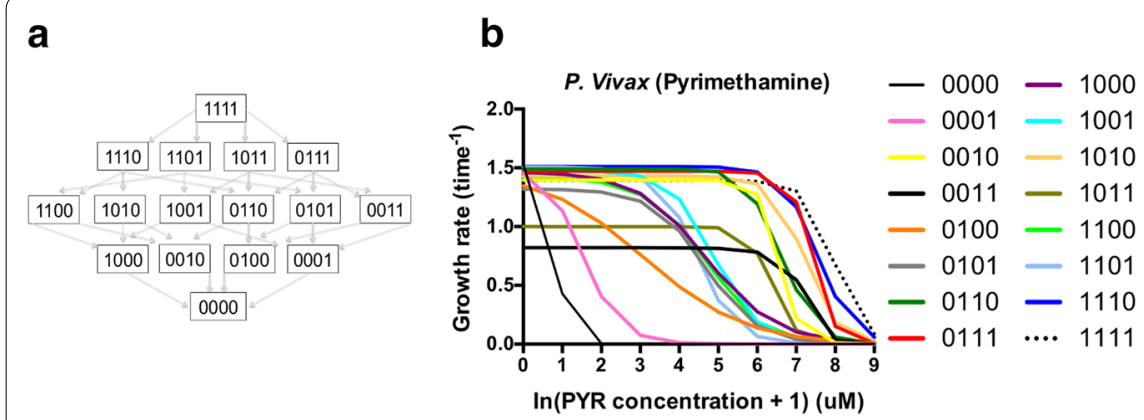

C

Rank order of allele out of the total set (16)

Fig. 1 Alleles composing the Plasmodium vivax adaptive landscape for drug resistance in this study. a Schematic of the possible pathways between the most resistant allele (1111) and most susceptible (0000). b Growth rates of alleles in the landscape of $P$. vivax as observed in Jiang et al. [32] c Rank order curves for P. vivax in pyrimethamine. The $y$-axis depicts the rank order of alleles at a given drug concentration. The $x$-axis is in terms In(concentration of PYR +1 ) of the pyrimethamine drug concentration in $\mu \mathrm{M}$. Note how regularly the lines intersect across drug concentrations. This indicates $G \times E$ interactions, which alters the structure of the landscape 


\section{Simulations of evolution}

Having identified the $\mathrm{S} 117 \mathrm{~N}$ mutation as having the greatest $\mathrm{G} \times \mathrm{E}$ effect, computer simulations were used to test whether these (or other) effects constrain evolution in certain directions. SimuPop was used as the simulation convention, an individual-based, Wright-Fisher, forward-time simulation model [53] similar to that described by Jiang et al. [32]. In this model, generations are discrete (non-overlapping), with an effective population size of 10,000. Mutation rates were determined by the relative rate matrix for $P$. vivax computed from data in Neafsey et al. [54]. For the purposes of converting the relative substitution rate matrix to a more realistic pergeneration rate matrix, mutation rates were divided by $10^{3}$ and then converted into amino acid substitution rates using the sum of substitution rates of nucleotides responsible for drug resistance in $P$. vivax DHFR.

The mutation rates were also scaled by a factor of 1000 to allow simulations with fewer individuals. Scaling involves dividing the population size by a scaling factor, $m$, and then multiplying the mutation rates by that same factor:

$$
N_{e} \cdot \mu=\frac{N_{e}}{m}(\mu \cdot m)
$$

The simulations were designed to simulate the dynamics of reverse evolution in two population genetic scenarios:

1. A population fixed for the most resistant (1111) allele for 1000 generations across a range of drug concentrations $(\sim 3000, \sim 400, \sim 55, \sim 7 \mu \mathrm{M})$. This allows one to observe the general dynamics of reverse evolution, and test whether reversion towards the wild type (0000) ever occurs. The most obvious prediction would be that at the extremely high PYR concentration $(\sim 3000 \mu \mathrm{M})$, the population should remain trapped on the 1111 allele, as it is the most resistant allele in the set and has the highest growth rate at the highest concentration (Fig. 1b, c, Additional file 3).

2. A population composed equally of all six double mutants (1100, 1010, 1001, 0011, 0101, 0110), evolving in the absence of drug. Because the double mutants are in the centre of the landscape (in terms of Hamming distance between 0000 and 1111), simulations with them as a starting point would uncover any intrinsic landscape bias towards forward or reverse evolution.

\section{Results}

The structure of reverse evolution trajectories

Using fitness values for P. vivax based on Eq. 1, threedimensional representations of all possible trajectories were constructed for each drug across several drug concentrations (Fig. 2). In particular, one should highlight the structure of the reverse evolutionary trajectories at the two lower drug concentrations (no drug and $\sim 7 \mu \mathrm{M}$, Fig. 2a, b), as the wild-type ancestor (0000) has a relatively high fitness in both (Fig. 1). Note the presence of fitness valleys in all trajectories between 1111 and 0000 , even at low concentrations, indicating that 0000 is inaccessible through mutation-selection balance alone. This characteristic of the trajectories is further examined in other parts of this study. Keep in mind that the quadruple mutant (1111) might not exist in nature for P. vivax DHFR. This means that this exact scenario might not reflect how reversal occurs in nature, but still changes little about the purpose or relevance of the study: to diagnose features of the adaptive landscape that explain why reverse evolution might be difficult, rather than explain any single finding in any particular ecological context. In order to do so, evolution was modelled from one extreme of the landscape (1111) to the other (0000), all towards a conceptual and mechanistic understanding of the constraints on reverse evolution, suggesting forces at play in wild populations of malaria parasite.

\section{Analysis of the fitness effects of mutations across drug concentrations reveals a single mutation of uncommonly large positive effect}

The fitness effect of individual mutations across drug concentrations was then measured. Figure 3a displays both the average effects (solid lines) and individual effect points (scattered points). Here, one can see that the third site mutation, S117N $\left(^{* * *} 1^{*}\right)$ has a strongly positive fitness effect across environments $\left(P=2.22 \times 10^{-8}\right.$, Additional file 4). To observe how this mutation contributes to the fitness of alleles composing the landscape, the average growth rate of all alleles that carry each individual mutation was then calculated (Fig. 3b). This analysis reveals that alleles containing the S117N mutation have significantly higher growth rates across environments than alleles carrying the other mutations $(P=0.025 ; \mathrm{F}=5.7 ; \mathrm{df}=3,36)$ (Fig. 3b).

\section{Simulations of evolution}

Next, evolution was simulated across the adaptive landscape to test whether $\mathrm{S} 117 \mathrm{~N}$ plays a key role in impeding reversal in two settings: starting from (1) a population composed of the most resistant allele (1111), and, (2) from the centre of the landscape with a population divided equally between each of the six double mutants (see "Methods"). Figures 4 and 5 depict illustrative dynamics of evolution in these simulations, and Table 1 contains a more detailed summary of all simulation runs.

Simulations demonstrate that populations fixed for the 1111 allele do not undergo reverse evolution to the 

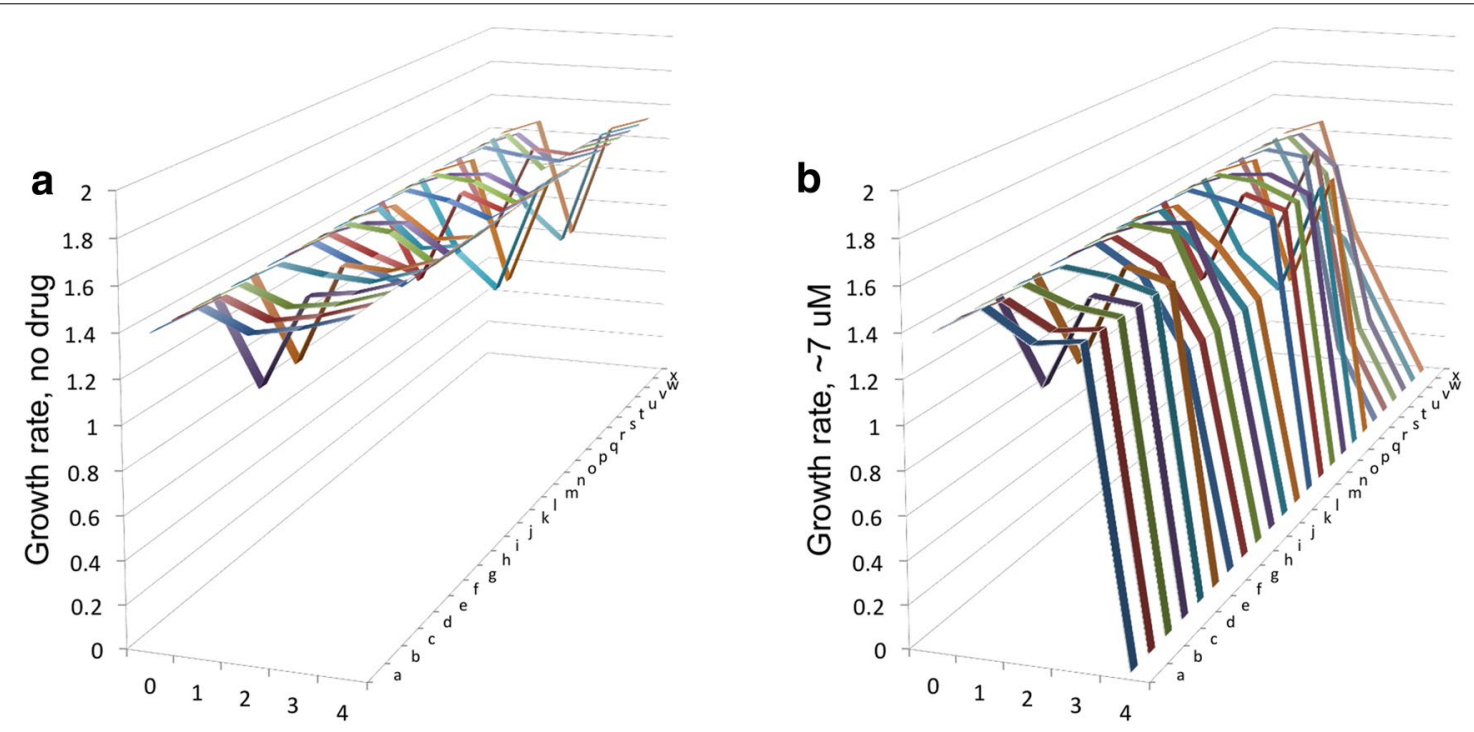

Hamming distance
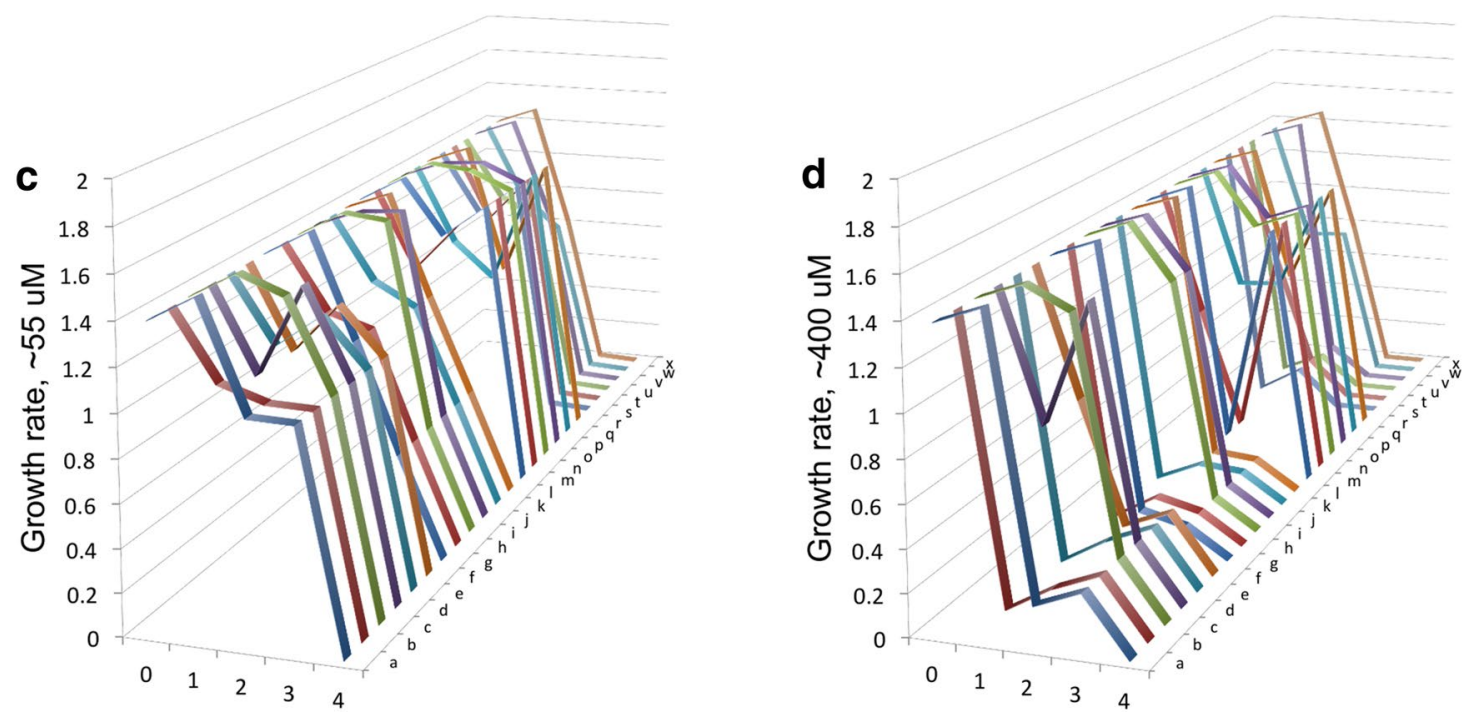

Fig. 2 The structure of reverse evolution trajectories in Plasmodium vivax. Twenty-four adaptive landscapes for P. vivax DHFR across several drug concentrations, organized into individual trajectories. The $y$-axis is growth rate. The $x$-axis denotes hamming distance from the original, which is the quadruple mutant (1111) in this study $(0=$ quadruple mutant, 1 triple mutant, 2 double mutant, 3 singe mutant, 4 ancestral allele), and the $z$-axis corresponds to the 24 different possible pathways between the most resistant allele (1111) and the ancestral allele (0000). Additional file 6 identifies the individual pathways $a-x$. Growth rates are in units of time $e^{-1}$. Concentrations: a no drug, b $\sim 7 \mu \mathrm{M}, \mathbf{c} \sim 55 \mu \mathrm{M}, \mathbf{d} \sim 400 \mu \mathrm{M}$

ancestral allele at any drug concentration (including the drugless environment) even after a thousand generations, but are trapped at the 1110 triple mutant fitness peak at most concentrations, with a small fraction of simulations leading to the 0111 triple mutant (Fig. 4; Table 1). This finding reflects the fact that the 1110 triple mutant has a high growth rate even in the drugless environment, superior to all of its double- and single-mutant neighbours. Although the 0110, 1110 and 0111 alleles (all of which contain the S117N mutation) have a growth rate lower than the ancestor (0000) in the no-drug environment, evolving populations are unable to cross the singlemutant $(1000,0100,0010,0001)$ valley necessary to reach the ancestral genotype, precluding reverse evolution (Fig. 5). This is because the combination of the S117N mutation and the second-site mutation, S58R $\left({ }^{* 1 * *}\right)$ has properties of an epistatic ratchet [25] that restricts reverse evolution: it is able to reproduce well enough at 


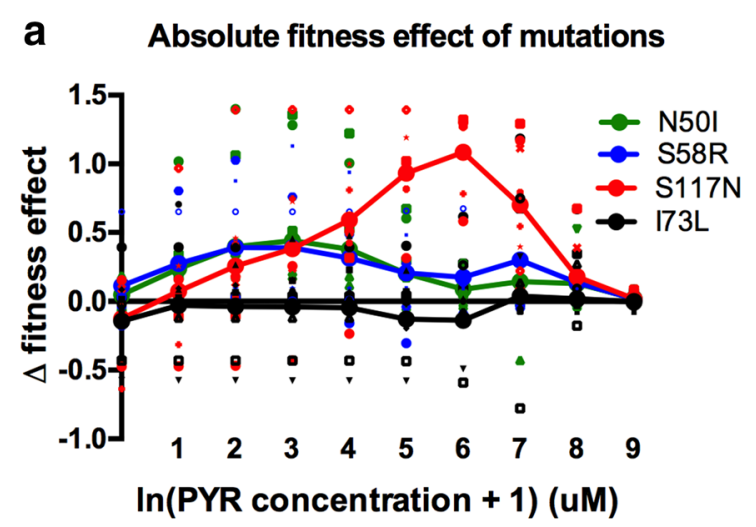

\section{b Growth rate of all alleles carrying a \\ given mutation}

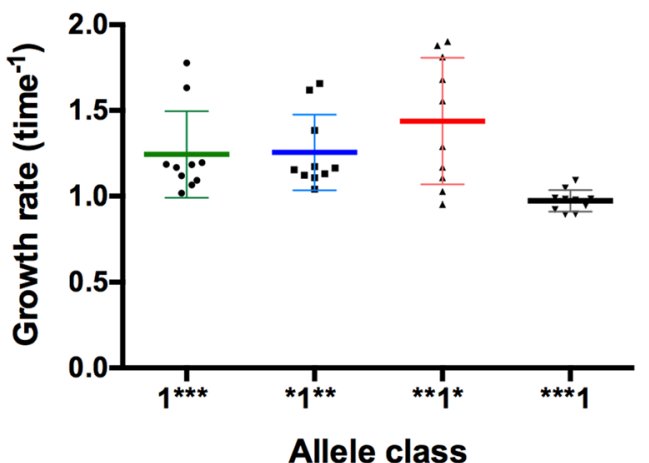

Fig. 3 A single mutation potentiator of $\mathrm{G} \times \mathrm{E}$ effects, S117N, has uncommonly high fitness effects across a range of drug concentrations, and creates alleles that are of higher fitness. a Each colour represents the $\Delta$ fitness effect of a one of the four mutations, the difference between a genotype with and without a mutation. Each mutation has 8 possible genetic backgrounds. Small (unconnected) symbols are representative of $\Delta$ fitness effect measures for individual mutation effects in a particular genetic background. Large symbols, connected by lines, represent the average $\Delta$ fitness effect of a mutation over all 8 genetic backgrounds. The third site (S117N) mutation has a strong effect, creating high fitness alleles that foster forward evolution and inhibit reverse evolution. The $x$-axis is in units In(concentration of PYR +1 ) $\mu M$. b Whole alleles carrying the $G \times E$ pivot mutation $\left({ }^{* *} 1^{*}\right.$ ) have significantly higher growth rates than the other alleles (averaged across drug concentrations). This graph differs from $\mathbf{a}$ because this does not depict fitness effects of single mutations, but rather, the total average fitness of whole alleles carrying the specified mutation
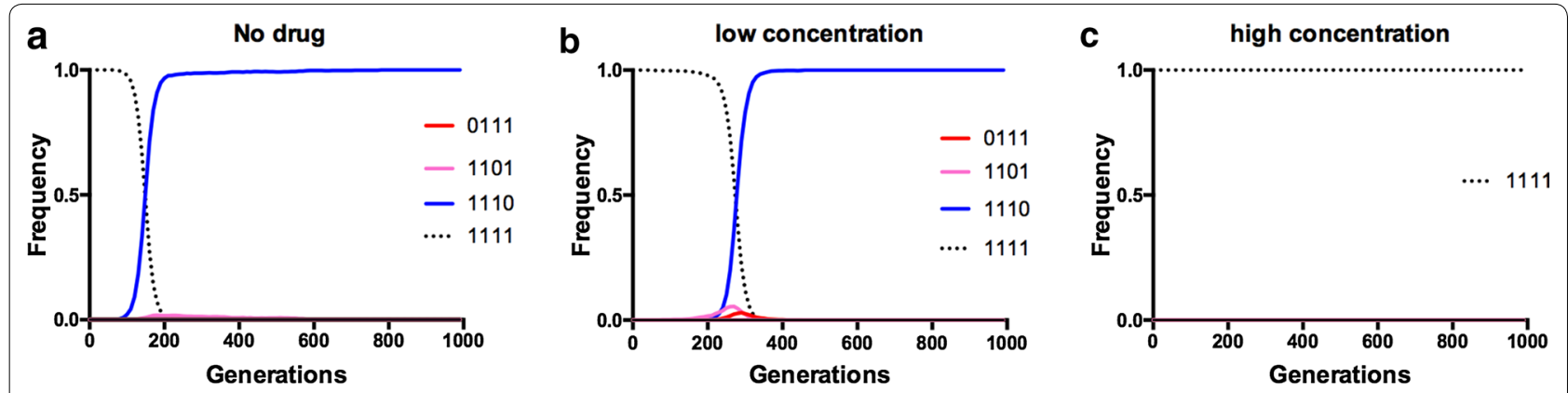

Fig. 4 Starting from the quadruple mutant (1111), reverse evolution towards the ancestor (0000) is impeded across concentrations. These are illustrative examples of the most preferred pathways for evolution at each of the simulated pyrimethamine environments, starting with the 1111 quadruple mutant. Panels correspond to several simulation scenarios: a no drug, b low drug $(\sim 7.0 \mu \mathrm{M})$, c high drug $(\sim 3000 \mu \mathrm{M})$. In each case, one observes no accessible trajectories in the fitness landscape leading to the ancestral allele (0000), not even in the no-drug environment where the ancestor is the most fit

both higher drug concentrations and in the drugless environment (Fig. 1b) to limit crossing the single mutation (0010 and 0100 in this case) valley necessary to reach the 0000 absolute fitness peak in the drugless environment.

\section{Conclusions}

While irreversibility across an adaptive landscape for antimicrobial resistance has been observed in many pathogen types, this question has been relatively unexplored in malarial parasites and in particular, as it pertains to a mechanism underlying this constraint. In the case of malaria, several past studies from the field, involving both chloroquine and pyrimethamine, support the assertion that reverse evolution is improbable: in one instance, a population of $P$. falciparum resistant to chloroquine reverted to wild type only after replacement with a migrant population composed of ancestral susceptible genotypes (rather than through de novo mutation and selection) [55]. In another, a population of P. falciparum resistant to pyrimethamine compensated through copy number variation in GTP cyclohydrolase in lieu of reversing the mutations already fixed in DHFR [56].

Although the study focused on P. vivax DHFR, it provides a conceptual basis for irreversibility in other resistance proteins. The findings from the field, in combination with these results, imply that modern whole-genome 


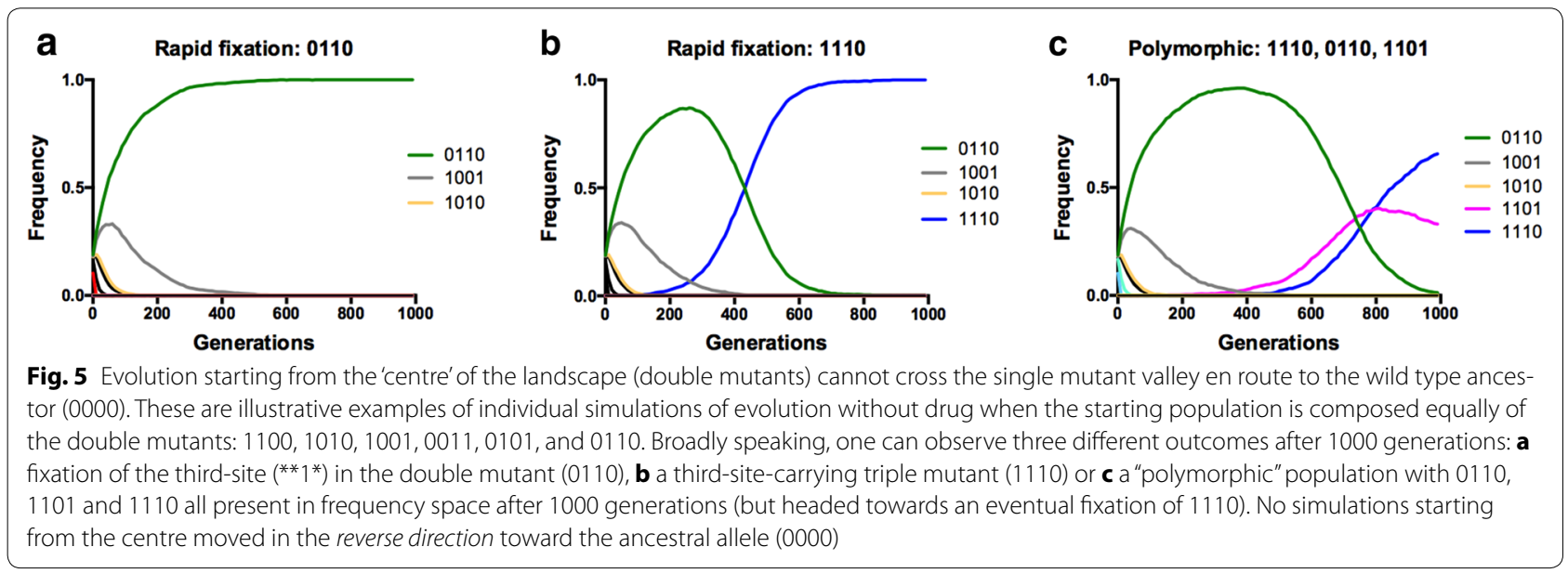

Table 1 Summary of simulations of evolution in two schemes

\begin{tabular}{|c|c|c|}
\hline \multicolumn{3}{|c|}{ (a) Reverse evolution simulation summary } \\
\hline Starting conditions (drug and allele) & Outcome & Fraction \\
\hline \multicolumn{3}{|l|}{$\sim 3000 \mu \mathrm{M}$ PYR (peak mutant = 1111) } \\
\hline 1111 & 1111 & 0.97 \\
\hline 1111 & $1111 \rightarrow 0111$ & 0.3 \\
\hline \multicolumn{3}{|l|}{$\sim 400$ MM PYR (peak mutant = 1110) } \\
\hline 1111 & $1111 \rightarrow 1110$ & 0.93 \\
\hline 1111 & $1111 \rightarrow 0111$ & 0.06 \\
\hline \multicolumn{3}{|l|}{$\sim 55 \mu \mathrm{M}$ PYR (peak mutant $=1110$ ) } \\
\hline 1111 & $1111 \rightarrow 1110$ & 0.96 \\
\hline 1111 & $1111 \rightarrow 0111 \rightarrow 0110$ & 0.04 \\
\hline \multicolumn{3}{|l|}{$\sim 7 \mu \mathrm{M}$ PYR (peak mutant $=1110$ ) } \\
\hline 1111 & $1111 \rightarrow 1110$ & 0.97 \\
\hline 1111 & $1111 \rightarrow 1101$ & 0.03 \\
\hline \multicolumn{3}{|l|}{ No drug (peak mutant $=0000$ ) } \\
\hline 1111 & $1111 \rightarrow 1110$ & 0.91 \\
\hline 1111 & $1111 \rightarrow 1101$ & 0.08 \\
\hline
\end{tabular}

(b) Double mutants, no drug simulation summary

Outcome

Equally distributed: 1100, 1001, 1010, 0011, 0101, 1110

$\begin{array}{rl}\text { Polymorphic } \rightarrow 1110 & 0.54 \\ \text { Polymorphic } \rightarrow 0110 & 0.27 \\ \text { Polymorphic } \rightarrow 1101 & 0.19\end{array}$

(a) Starting from a population fixed for 1111, across drug concentrations and (b) starting from the centre of the landscape, a population composed equally of the six double mutants. Note that this summary includes several drug concentrations not visualized in Figs. 4 and 5 . For (a) which allele in the landscape is the absolute peak at that drug concentration is also highlighted (in parentheses). In a smooth landscape, the landscape should be able to locate the absolute peak. Note that in the no drug environment, the population remains trapped on triple mutant local peaks (1110 and 1101), and unable to locate the 0000 absolute peak

sequencing efforts will reveal a more complex genomic signature of resistance and reversal in settings where antimicrobial use has waxed and waned. This has implications for the practice of molecular epidemiology: while sequencing selected SNPs in resistance determinants might be sufficient for identifying resistance alleles in settings where populations of pathogen are 'forward' evolving resistance, the genomic picture is likely more complicated in reverse. The results, corroborated by findings from the field, suggest that re-evolution of the 
susceptible phenotype (without the growth defects of the more resistant phenotypes) is likely to occur either through the introduction of susceptible migrant genotypes from elsewhere or compensatory mutations at sites other than the ones originally arising during resistance evolution $[57,58]$.

This study dissected barriers to reverse evolution from the most resistant genotype (1111) toward the most susceptible (0000) across an adaptive landscape for drug resistance mediated by DHFR in $P$. vivax. Among four amino acid replacements resulting in pyrimethamine resistance, a single site, S117N (**1*) had a strong effect on the fitness of alleles in the landscape across a breadth of drug concentrations. At high drug concentrations, double and triple mutants containing the S117N mutation, and in particular those in combination with the second site mutation S58R (0110 and 1110, for example) have a reproductive advantage across most drug environments. More specifically, while these higher order alleles have lower fitness than the 0000 ancestral allele in the absence of drug, they have substantially higher fitness than the single mutant neighbours that separate the higher-order mutants carrying the S117N (**1*) mutation (1000, 0100, 0010, 0001), which explains the low likelihood of reverse evolution across this drug resistance adaptive landscape.

Simulations of evolution across the landscape demonstrate the consequences of genotype-by-environment interactions involving S117N: whereas past studies have shown that forward evolution from the 0000 ancestor to an absolute fitness peak occurs readily at drug concentrations greater than 0 [32], evolution starting from the population fixed for the 1111 quadruple mutant becomes trapped at the 1110 triple mutant local fitness peak, even in the drugless environment (Fig. 4). Even more, when the landscape starts with a population distributed equally between the double mutants (the centre of the landscape; 0011, 0101, 1001, 1010, 0110, 1100), the evolutionary dynamics are still driven by the S117N site $\left(^{* * *} 1 *\right)$, usually resulting in fixation of the 1110 triple mutant (Fig. 5; Table 1). In this sense, the $\mathrm{S} 117 \mathrm{~N}$ mutation serves as a pivot point for mutation: its arrival provides a bridge to high fitness areas of the landscape that are trapped onto local peaks through their interaction with other mutations, unable to move to other areas of the landscape.

These findings support the existence of epistatic ratchets that inhibit reverse evolution towards ancestral states, such as that observed in the evolution of the vertebrate glucocorticoid receptor [25]. While the pivotal S117N mutation creates a ratchet through epistatic interactions, its average effect alone (across all genetic backgrounds and across environments) is larger than that of the other sites $\left(1^{* * *}, 1^{* * * * * * 1)}\right.$, indicating that its fitness effects are not limited to singular genetic backgrounds or certain environments. In this sense, S117N opens evolutionary 'forks in the road' towards higher mutation regions of the landscape (double mutants, triple mutants and the quadruple mutant), serving as the starting material for the epistatic ratchets that ultimately prevent reverse evolution.

Other than the implications for molecular epidemiology discussed above, these findings are most relevant to debates surrounding best practices for antimicrobial resistance management. The notion that ceasing use of antimicrobials is a viable strategy for decreasing resistance is based, in part, on the assumption that reverse evolution can occur across a landscape because of the fitness cost of resistance. The results suggest that such strategies may not be generally valid, and they should be tailored to the nature of the actual adaptive landscape and its $G \times E$ interactions affecting the accessible trajectories towards resistance and susceptibility. Please note that these comments apply to stepwise reverse evolution in a situation where a derived resistance allele (1111 in this manuscript) is fixed. Alternatively, if a population retains the ancestral allele at low frequency, it can increase in frequency in the absence of drug. This latter scenario is not stepwise evolution, however, but canonical selection on standing genetic variation, a different population genetic context than the one simulated in this study.

Lastly, and most provocatively, the identification of the S117N pivot mutation suggests a possible strategy for identifying targets for chemotherapeutic intervention: if a single mutation is a pivot point to large fitness effects (as found in this study), it might be an ideal drug target. Compounds that perturb the interaction between these pivot residues and others might have a destabilizing effect on protein structure or function, and diminish the evolutionary potential of alleles carrying the resistance determinant. Because this strategy would target the ability of a protein to reach high fitness areas of adaptive landscapes, it would constitute a strategy directed against the evolvability of the pathogen, an unexplored avenue for the treatment of microbial pathogens. 


\section{Additional files}

Additional file 1: Table S1. Values and standard errors for the empirical derived parameters used to model growth rates: Drugless growth rates, IC50 values.

Additional file 2: Table S2. Standardized simulated growth rates as depicted in Fig. 1 b.

Additional file 3: Table S3. The rank order of alleles as depicted in Fig. 1.

Additional file 4: Table S4. ANOVA: Interaction between mutation effect and drug concentration for Plasmodium vivax in the presence of pyrimethamine. All values for dfnum.,denom. =9, 70 .

Additional file 5: Figure S1. Standard deviation of the absolute fitness effects of a mutation (epistasis). Epistasis can be measured any number of ways, however, the standard deviation provides a proxy: it measures the dispersion of $\mathrm{G} \times \mathrm{G}$ effects for a given mutation at a given drug.

Additional file 6: Table S5. Discrete pathways with letter corresponding to the paths in Fig. 2.

\section{Authors' contributions}

$\mathrm{CBO}$ and DLH designed the study. CBO carried out the calculations and computer simulations. $\mathrm{CBO}$ analysed the results. $\mathrm{CBO}$ and $\mathrm{DLH}$ wrote the manuscript. Both authors read and approved the final manuscript.

\section{Author details}

${ }^{1}$ Department of Biology, University of Vermont, Burlington, VT, USA. ${ }^{2}$ Vermont Complex Systems Center, The University of Vermont, Burlington, VT, USA. ${ }^{3}$ Department of Organismic and Evolutionary Biology, Harvard University, Cambridge, MA, USA.

\section{Acknowledgements}

The authors would like to thank Pan Pan Jiang for discussions on the dataset, and Russell Corbett-Detig for his input on the simulations. This work was supported by NIH Grant Al106734 to DLH. Support for CBO comes from the George Washington Henderson Fellowship at the University of Vermont and by the Ford Foundation Postdoctoral Fellowship. The authors thank C Scott Wylie, Daniel Weinreich, Seth Rakoff-Nahoum and Rachel Rutishauser for helpful discussions.

\section{Competing interests}

The authors declare that they have no competing interests.

Received: 12 September 2015 Accepted: 10 January 2016

Published online: 25 January 2016

\section{References}

1. Read AF, Day T, Huijben S. The evolution of drug resistance and the curious orthodoxy of aggressive chemotherapy. Proc Natl Acad Sci USA. 2011;108(Suppl 2):10871-7.

2. Huijben S, Bell AS, Sim DG, Tomasello D, Mideo N, Day T, et al. Aggressive chemotherapy and the selection of drug resistant pathogens. PLoS Pathog. 2013;9:e1003578.

3. Kouyos RD, Metcalf CJE, Birger R, Klein EY, Abel zur Wiesch P, Ankomah P, et al. The path of least resistance: aggressive or moderate treatment? Proc Biol Sci. 2014;281:20140566.

4. Carlet J, Jarlier V, Harbarth S, Voss A, Goossens H, Pittet D, et al. Ready for a world without antibiotics? The pensières antibiotic resistance call to action. Antimicrob Resist Infect Control. 2012;1:11.

5. Kaki R, Elligsen M, Walker S, Simor A, Palmay L, Daneman N. Impact of antimicrobial stewardship in critical care: a systematic review. J Antimicrob Chemother. 2011;66:1223-30.

6. Dellit TH, Owens RC, McGowan JE, Gerding DN, Weinstein RA, Burke JP, et al. Infectious Diseases Society of America and the Society for Healthcare Epidemiology of America guidelines for developing an institutional program to enhance antimicrobial stewardship. Clin Infect Dis. 2007:44:159-77.

7. Antibiotic Resistance Threats in the United States. 2013. http://www.cdc gov/drugresistance/threat-report-2013/.

8. Bergstrom CT, Lo M, Lipsitch M. Ecological theory suggests that antimicrobial cycling will not reduce antimicrobial resistance in hospitals. Proc Natl Acad Sci USA. 2004;101:13285-90.

9. Goulart CP, Mahmudi M, Crona KA, Jacobs SD, Kallmann M, Hall BG, et al. Designing antibiotic cycling strategies by determining and understanding local adaptive landscapes. PLoS One. 2013;8:e56040.

10. Toprak E, Veres A, Michel J-B, Chait R, Hartl DL, Kishony R. Evolutionary paths to antibiotic resistance under dynamically sustained drug selection. Nat Genet. 2012:44:101-5.

11. Kim S, Lieberman TD, Kishony R. Alternating antibiotic treatments constrain evolutionary paths to multidrug resistance. Proc Natl Acad Sci. 2014;111:14494-9.

12. Imamovic L, Sommer MOA. Use of collateral sensitivity networks to design drug cycling protocols that avoid resistance development. Sci Transl Med. 2013;5:204ra132.

13. AbelZurWiesch P, Kouyos R, Abel S, Viechtbauer W, Bonhoeffer S. Cycling empirical antibiotic therapy in hospitals: meta-analysis and models. PLoS Pathog. 2014;10:e1004225.

14. Mölstad S, Erntell M, Hanberger H, Melander E, Norman C, Skoog G, et al. Sustained reduction of antibiotic use and low bacterial resistance: 10-year follow-up of the Swedish Strama programme. Lancet Infect Dis. 2008:8:125-32.

15. Seppälä H, Klaukka T, Vuopio-Varkila J, Muotiala A, Helenius H, Lager K, et al. The effect of changes in the consumption of macrolide antibiotics on erythromycin resistance in group A streptococci in Finland. Finnish Study Group for Antimicrobial Resistance. N Engl J Med. 1997;337:441-6.

16. Sjölund M, Wreiber K, Andersson DI, Blaser MJ, Engstrand L. Long-term persistence of resistant Enterococcus species after antibiotics to eradicate Helicobacter pylori. Ann Intern Med. 2003;139:483-7.

17. Andersson DI, Hughes D. Persistence of antibiotic resistance in bacterial populations. FEMS Microbiol Rev. 2011;35:901-11.

18. Andersson DI, Hughes D. Antibiotic resistance and its cost: is it possible to reverse resistance? Nat Rev Microbiol. 2010;8:260-71.

19. Sundqvist M, Geli P, Andersson DI, Sjölund-Karlsson M, Runehagen A, Cars $\mathrm{H}$, et al. Little evidence for reversibility of trimethoprim resistance after a drastic reduction in trimethoprim use. J Antimicrob Chemother. 2010;65:350-60

20. Handel A, Regoes RR, Antia R. The role of compensatory mutations in the emergence of drug resistance. PLoS Comput Biol. 2006;2:e137.

21. Levin BR, Perrot V, Walker N. Compensatory mutations, antibiotic resistance and the population genetics of adaptive evolution in bacteria. Genetics. 2000;154:985-97.

22. Gould SJ. Dollo on Dollo's law: irreversibility and the status of evolutionary laws. J Hist Biol. 1970;3:189-212.

23. Dawkins R. The blind watchmaker: why the evidence of evolution reveals a universe without design. New York: WW. Norton \& Company; 1996. p 496

24. Tan L, Serene S, Chao HX, Gore J. Hidden randomness between fitness landscapes limits reverse evolution. Phys Rev Lett. 2011;106:198102.

25. Choowongkomon K, Ortlund EA, Thornton JW. An epistatic ratchet constrains the direction of glucocorticoid receptor evolution. Nature. 2009:461:515-9.

26. WHO. World Malaria Report 2014. Geneva, World Health Organization, 2014. http://www.who.int/malaria/publications/ world_malaria_report_2014/en/.

27. Weinreich DM, Delaney NF, Depristo MA, Hartl DL. Darwinian evolution can follow only very few mutational paths to fitter proteins. Science. 2006;312:111-4

28. Poelwijk FJ, Kiviet DJ, Weinreich DM, Tans SJ. Empirical fitness landscapes reveal accessible evolutionary paths. Nature. 2007;445:383-6.

29. Lozovsky ER, Chookajorn T, Brown KM, Imwong M, Shaw PJ, Kamchonwongpaisan S, et al. Stepwise acquisition of pyrimethamine resistance in the malaria parasite. Proc Natl Acad Sci USA. 2009;106:12025-30.

30. Brown KM, Costanzo MS, Xu W, Roy S, Lozovsky ER, Hartl DL. Compensatory mutations restore fitness during the evolution of dihydrofolate reductase. Mol Biol Evol. 2010;27:2682-90.

31. Costanzo MS, Hartl DL. The evolutionary landscape of antifolate resistance in Plasmodium falciparum. J Genet. 2011;90:187-90. 
32. Jiang P-P, Corbett-Detig RB, HartI DL, Lozovsky ER. Accessible mutational trajectories for the evolution of pyrimethamine resistance in the malaria parasite Plasmodium vivax. J Mol Evol. 2013;77:81-91.

33. de Pécoulas PE, Tahar R, Ouatas T, Mazabraud A, Basco LK. Sequence variations in the Plasmodium vivax dihydrofolate reductase-thymidylate synthase gene and their relationship with pyrimethamine resistance. Mol Biochem Parasitol. 1998;92:265-73.

34. Miao M, Yang Z, Cui L, Ahlum J, Huang Y, Cui L. Different allele prevalence in the dihydrofolate reductase and dihydropteroate synthase genes in Plasmodium vivax populations from China. Am J Trop Med Hyg 2010;83:1206-11.

35. Imwong M, Pukrittakayamee S, Looareesuwan S, Pasvol G, Poirreiz J, White NJ, et al. Association of genetic mutations in Plasmodium vivax dhfr with resistance to sulfadoxine-pyrimethamine: geographical and clinical correlates. Antimicrob Agents Chemother. 2001;45:3122-7.

36. Mint Lekweiry K, Ould Mohamed Salem Boukhary A, Gaillard T, Wurtz N, Bogreau $\mathrm{H}$, Hafid JE, et al. Molecular surveillance of drug-resistant Plasmodium vivax using pvdhfr, pvdhps and pvmdr1 markers in Nouakchott, Mauritania. J Antimicrob Chemother. 2012;67:367-74.

37. Afsharpad M, Zakeri S, Pirahmadi S, Djadid ND. Molecular assessment of dhfr/dhps mutations among Plasmodium vivax clinical isolates after introduction of sulfadoxine/pyrimethamine in combination with artesunate in Iran. Infect Genet Evol. 2012;12:38-44.

38. Khatoon L, Baliraine FN, Bonizzoni M, Malik SA, Yan G. Prevalence of antimalarial drug resistance mutations in Plasmodium vivax and $P$. falciparum from a malaria-endemic area of Pakistan. Am J Trop Med Hyg. 2009:81:525-8.

39. Barnadas C, Musset L, Legrand E, Tichit M, Briolant S, Fusai T, et al. High prevalence and fixation of Plasmodium vivax dhfr/dhps mutations related to sulfadoxine/pyrimethamine resistance in French Guiana. Am J Trop Med Hyg. 2009;81:19-22.

40. Lu F, Lim CS, Nam DH, Kim K, Lin K, Kim T-S, et al. Mutations in the antifolate-resistance-associated genes dihydrofolate reductase and dihydropteroate synthase in Plasmodium vivax isolates from malaria-endemic countries. Am J Trop Med Hyg. 2010;83:474-9.

41. Schunk M, Kumma WP, Miranda IB, Osman ME, Roewer S, Alano A, et al. High prevalence of drug-resistance mutations in Plasmodium falciparum and Plasmodium vivax in southern Ethiopia. Malar J. 2006;5:54.

42. Barnadas C, Ratsimbasoa A, Tichit M, Bouchier C, Jahevitra M, Picot S, et al. Plasmodium vivax resistance to chloroquine in Madagascar: clinical efficacy and polymorphisms in pvmdr1 and pvcrt-o genes. Antimicrob Agents Chemother. 2008;52:4233-40.

43. Brega S, de Monbrison F, Severini C, Udomsangpetch R, Sutanto I, Ruckert $P$, et al. Real-time PCR for dihydrofolate reductase gene single-nucleotide polymorphisms in Plasmodium vivax isolates. Antimicrob Agents Chemother. 2004;48:2581-7.

44. Valecha N, Joshi H, Eapen A, Ravinderan J, Kumar A, Prajapati SK, et al. Therapeutic efficacy of chloroquine in Plasmodium vivax from areas with different epidemiological patterns in India and their Pvdhfr gene mutation pattern. Trans R Soc Trop Med Hyg. 2006;100:831-7.

45. Hastings IM, Donnelly MJ. The impact of antimalarial drug resistance mutations on parasite fitness, and its implications for the evolution of resistance. Drug Resist Updat Rev Comment Antimicrob Anticancer Chemother. 2005;8:43-50.
46. Auliff A, Wilson DW, Russell B, Gao Q, Chen N, Le Ngoc A, et al. Amino acid mutations in Plasmodium vivax DHFR and DHPS from several geographical regions and susceptibility to antifolate drugs. Am J Trop Med Hyg. 2006;75:617-21.

47. Peng B, Amos Cl, Kimmel M. Forward-time simulations of human populations with complex diseases. PLoS Genet. 2007;3:e47.

48. Lipka B, Milewska-Bobula B, Filipek M. Monitoring of plasma concentration of pyrimethamine (PYR) in infants with congenital Toxoplasma gondii infection—own observations. Wiad Parazytol. 2011;57:87-92.

49. Dzinjalamala FK, Macheso A, Kublin JG, Taylor TE, Barnes KI, Molyneux ME, et al. Blood folate concentrations and in vivo sulfadoxine-pyrimethamine failure in Malawian children with uncomplicated Plasmodium falciparum malaria. Am J Trop Med Hyg. 2005;72:267-72.

50. Dzinjalamala FK, Macheso A, Kublin JG, Taylor TE, Barnes KI, Molyneux ME, et al. Association between the pharmacokinetics and in vivo therapeutic efficacy of sulfadoxine-pyrimethamine in Malawian children. Antimicrob Agents Chemother. 2005;49:3601-6.

51. Jamaludin A, Mohamad M, Navaratnam V, Yeoh PY, Wernsdorfer WH Multiple-dose pharmacokinetic study of proguanil and cycloguanil following 12-hourly administration of $100 \mathrm{mg}$ proguanil hydrochloride. Trop Med Parasitol. 1990;41:268-72.

52. Weidekamm E, Plozza-Nottebrock H, Forgo I, Dubach UC. Plasma concentrations of pyrimethamine and sulfadoxine and evaluation of pharmacokinetic data by computerized curve fitting. Bull World Health Organ. 1982;60:115-22

53. Weinreich DM, Lan Y, Wylie CS, Heckendorn RB. Should evolutionary geneticists worry about higher-order epistasis? Curr Opin Genet Dev. 2013;23:700-7.

54. Neafsey DE, Galinsky K, Jiang RHY, Young L, Sykes SM, Saif S, et al. The malaria parasite Plasmodium vivax exhibits greater genetic diversity than Plasmodium falciparum. Nat Genet. 2012;44:1046-50.

55. Kublin JG, Cortese JF, Njunju EM, Mukadam RAG, Wirima JJ, Kazembe PN, et al. Reemergence of chloroquine-sensitive Plasmodium falciparum malaria after cessation of chloroquine use in Malawi. J Infect Dis. 2003; 187:1870-5.

56. Heinberg A, Siu E, Stern C, Lawrence EA, Ferdig MT, Deitsch KW, et al. Direct evidence for the adaptive role of copy number variation on antifolate susceptibility in Plasmodium falciparum. Mol Microbiol. 2013;88:702-12

57. Kümpornsin K, Kotanan N, Chobson P, Kochakarn T, Jirawatcharadech $P$, Jaru-ampornpan P, et al. Biochemical and functional characterization of Plasmodium falciparum GTP cyclohydrolase I. Malar J. 2014;13:150.

58. Pelleau S, Moss EL, Dhingra SK, Volney B, Casteras J, Gabryszewski SJ, et al. Adaptive evolution of malaria parasites in French Guiana: reversal of chloroquine resistance by acquisition of a mutation in pfert. Proc Natl Acad Sci USA. 2015;112:11672-7.

\section{Submit your next manuscript to BioMed Central and we will help you at every step:}

- We accept pre-submission inquiries

- Our selector tool helps you to find the most relevant journal

- We provide round the clock customer support

- Convenient online submission

- Thorough peer review

- Inclusion in PubMed and all major indexing services

- Maximum visibility for your research

Submit your manuscript at www.biomedcentral.com/submit

(O) BioMmed Central 